\title{
BARRELLED SUBSPACES OF SPACES WITH SUBSERIES DECOMPOSITIONS OR BOOLEAN RINGS OF PROJECTIONS
}

\author{
by LECH DREWNOWSKI, MIGUEL FLORENCIO and PEDRO J. PAÚL
}

(Received 26 June, 1992)

1. Introduction and basic general facts. The research presented in this paper started by extending a theorem of Swetits [18] about barrelledness of subspaces of metrizable AK-spaces to general AK-spaces of scalar sequences. The extension reads as follows.

(1) A subspace $\lambda_{0}$ of a barrelled $A K$-space $\lambda$ such that $\lambda_{0} \supset \phi$ is barrelled if and only if its dual $\lambda_{0}^{\prime}$ is weak ${ }^{*}$ sequentially complete. If in addition $\lambda_{0}$ is monotone, then it is barrelled if and only if $\lambda_{0}^{\prime}$ equals the Köthe dual $\lambda_{0}^{\times}$of $\lambda_{0}$.

As an easy consequence of this extension, we obtained the following result of Elstrodt and Roelcke [8, Corollary 3.4].

(2) If $\lambda$ is a barrelled monotone AK-space, then also its subspace $\mathscr{Z}(\lambda)$, consisting of all sequences in $\lambda$ with zero-density support, is barrelled.

At this point it was natural to ask whether the AK hypothesis was essential here; in particular, we were interested in knowing if the space $\mathscr{L}\left(l^{\infty}\right)$ was barrelled. We approached this problem as follows. We first established that the Boolean ring $\mathscr{Z}$ consisting of the subsets of $\mathbb{N}$ with density zero has Property (N); that is, the Nikodym uniform boundedness theorem holds for bounded finitely additive measures on $\mathscr{Z}$. This curious fact is of crucial importance for us, and it should also be of independent interest for people working in measure theory because the ring $\mathscr{Z}$ fails to satisfy any known conditions that ensure Property $(\mathrm{N})$. Using it, along with the obvious fact that the projections $P_{A}\left(x_{n}\right)=\left(\chi_{A}(n) \cdot x_{n}\right)(A \in \mathscr{L})$ form an equicontinuous Boolean ring of projections in $l^{\infty}$, we could extend the result (2) above to the case when $\lambda=l^{\infty}$. Very soon it turned out that, actually, the last result is valid in even much greater generality, namely for any barrelled locally convex space $E$ with an equicontinuous Boolean ring of projections $\mathbf{P}_{\mathscr{R}}$, where the "representing" ring of sets $\mathscr{R}$ has Property (N). This is presently the contents of Section 3 of our paper.

Since these results are valid for spaces more general than sequence spaces, it became desirable to achieve some sort of conformity with our earlier results (1) and (2) given for sequence AK-spaces. We decided, therefore, to leave the territory of scalar sequence spaces, and to generalize those earlier results to locally convex spaces with Schauder (or Markushevich) decompositions. This is done in Section 2 of our paper. In short, our main results are Theorem 1 in Section 2 and Theorem 2 in Section 3, and they are broad generalizations of the above results (1) and (2), respectively.

We refer the reader to [12], [14] or [20] for the terminology concerning locally convex spaces. However, we recall here the notion of Banach-Mackey spaces and two general facts.

Let $E$ be a (Hausdorff) locally convex space. Then, as usual, $E^{\prime}$ denotes the topological dual of $E$, and $E^{*}$ the algebraic dual of $E$. The space $E$ is said to be Banach-Mackey [20, p. 158] if it has the following uniform boundedness property: every subset of $E^{\prime}$ that is pointwise bounded on $E$ is uniformly bounded on every bounded subset of $E$. In other words, the requirement is that every $\sigma\left(E^{\prime}, E\right)$-bounded subset of $E^{\prime}$

Glasgow Math. J. 36 (1994) 57-69. 
is $\beta\left(E^{\prime}, E\right)$-bounded. Of course, the name for this class of spaces comes from the well-known Banach-Mackey Theorem asserting that a sequentially complete space $E$ has always the above property.

We will need below the following two facts concerning barrelled and quasi-barrelled locally convex spaces. For Fact A, see e.g. [14, Ch. 4 and 5] or [20, Problem 10-4-108 on p. 161]. Since we have been unable to locate a reference for Fact B (which is certainly well-known), we include a proof for the sake of completeness.

FACT A. For a locally convex space $E$ the following are equivalent.

(a) $E$ is barrelled.

(b) $E$ is quasi-barrelled and $E^{\prime}$ is $\sigma\left(E^{\prime}, E\right)$-sequentially complete.

(c) $E$ is quasi-barrelled and Banach-Mackey.

FACT B. A sequentially dense subspace $F$ of any barrelled locally convex space $E$ is quasi-barrelled.

Proof. Identifying $F^{\prime}$ with $E^{\prime}$, it is enough to show that if a set $M \subset E^{\prime}$ is $\beta\left(E^{\prime}, F\right)$-bounded, then it is $\sigma\left(E^{\prime}, E\right)$-bounded. Let $x \in E$. Then there is a sequence $\left(x_{n}\right)$ in $F$ converging to $x$. By assumption, $M$ is bounded on the bounded set $\left\{x_{n}: n \in \mathbb{N}\right\} \subset F$; thus $K:=\sup \left\{\left|\left\langle u, x_{n}\right\rangle\right|: u \in M, n \in \mathbb{N}\right\}<\infty$. Since $\left\langle u, x_{n}\right\rangle \rightarrow\langle u, x\rangle$ as $n \rightarrow \infty$ for every $u \in M$, it follows that $\sup \{|\langle u, x\rangle|: u \in M\} \leqslant K<\infty$.

COROLlaRY 1. A sequentially dense subspace $F$ of a barrelled locally convex space $E$ is barrelled if and only if $\left(E^{\prime}, \sigma\left(E^{\prime}, F\right)\right) \equiv\left(F^{\prime}, \sigma\left(F^{\prime}, F\right)\right)$ is sequentially complete.

2. Barrelled subspaces of spaces with subseries decompositions. As we mentioned in the introduction, the results in this section will be given for spaces with decompositions, in particular, sequence spaces. If one writes using as little terminology as possible, the main general results (Corollaries 2 and 4 ) can be stated as follows.

THEOREM 1. Let $E$ be a barrelled locally convex space with a Schauder decomposition $\left(P_{n}\right)$. Let $F$ be a subspace of $E$ containing all the summands $E_{n}:=P_{n}(E)(n \in \mathbb{N})$. Then $F$ is barrelled if and only if $\left(E^{\prime}, \sigma\left(E^{\prime}, F\right)\right)$ is sequentially complete.

Moreover, if for every $x \in F$ its expansion $\sum_{n=1}^{\infty} P_{n} x$ is subseries convergent in $F$, then the following are equivalent.

(a) $F$ is barrelled.

(b) If a sequence $u=\left(u_{n}\right) \in \prod_{n \in \mathbb{N}} E_{n}^{\prime}$ is such that the series $\langle u, x\rangle:=\sum_{n=1}^{\infty}\left\langle u_{n}, P_{n} x\right\rangle$ converges for all $x \in F$, then it also converges for all $x \in E$ (or, equivalently, the linear functional $u$ defined on $F$ by the formula above is continuous).

For the sake of clarity, and also to facilitate the comparison, we now review briefly some of the basic concepts and facts of the theory of sequence spaces, and some standard terminology used for spaces with decompositions.

Terminology for Sequence Spaces. Following [11] or [15], $\omega$ stands for the vector space of all sequences of (real or complex) numbers, and $\phi$ for the space of finitely non-zero sequences. A sequence space (or $K$-space) $\lambda$ is a linear subspace of $\omega$ that contains $\phi$ and is endowed with a locally convex topology finer than the topology of 
coordinate-wise convergence. Let $\lambda$ be a sequence space. We say that $\lambda$ is an $A K$-space if the sequence $\left(e_{n}\right)$ of unit vectors is a Schauder basis in this space. The space $\lambda$ is said to be monotone (resp. solid) if $\left(x_{n} z_{n}\right)$ is in $\lambda$ whenever $x=\left(x_{n}\right) \in \lambda$ and $\left(z_{n}\right)$ is a sequence of zeros and ones (resp. $\left|z_{n}\right| \leqslant 1$, for all $n \in \mathbb{N}$ ). The $\beta$-dual of $\lambda$ is defined as the space $\lambda^{\beta}$ of all sequences $y=\left(y_{n}\right) \in \omega$ such that the series $\sum_{n=1}^{\infty} x_{n} y_{n}$ converges for all $x=\left(x_{n}\right) \in \lambda$. When the ordinary convergence of the series in this definition is replaced by the absolute convergence, we obtain the space $\lambda^{\times}$, the well-known $\alpha$-dual or Köthe dual of $\lambda$. When $\lambda$ is monotone, these duals $\lambda^{\beta}$ and $\lambda^{\times}$coincide. In this way we obtain dual pairs $\left(\lambda, \lambda^{\beta}\right)$ and $\left(\lambda, \lambda^{\times}\right)$, with the obvious duality mapping $\langle x, y\rangle=\sum_{n=1}^{\infty} x_{n} y_{n}$. Consequently, the spaces $\lambda^{\beta}$ and $\lambda^{\times}$are usually treated as spaces of linear functionals: $\lambda^{\times} \subset \lambda^{\beta} \subset \lambda^{*}$. In particular, note that $\lambda^{\prime} \subset \lambda^{\beta}$ if $\lambda$ is an AK-space.

Terminology for Spaces with Decompositions. Let $E$ be a locally convex space. Following Singer [17, 15.20 on p. 514], an M-decomposition ("M" after Markushevich) of $E$ is a total sequence $\left(P_{n}\right)$ of pairwise orthogonal continuous linear projections in $E$. (Total means that if $P_{n} x=0$ for all $n$, then $x=0$.) The closed subspaces $E_{n}:=P_{n}(E)$ are called the summands of the decomposition. Any space $E$ with an M-decomposition may be viewed algebraically as a space of vector-valued sequences; namely, as a subspace of the product space $\prod_{n \in \mathbb{N}} E_{n}$, via the linear isomorphic embedding $x \mapsto\left(P_{n} x\right)$. Every sequence space has an obvious M-decomposition.

Throughout the remainder of this section, $E$ is a locally convex space with a fixed M-decomposition $\left(P_{n}\right)$ with summands $\left(E_{n}\right)$.

The space $E$ (or the decomposition) is said to be monotone if for every $x \in E$ and $A \subset \mathbb{N}$ there exists a (unique!) $x_{A} \in E$ such that $P_{n}\left(x_{A}\right)=P_{n} x$ for $n \in A$, and 0 otherwise. Of course, then the mappings $P_{A}: x \mapsto x_{A}$ thus obtained are linear projections in $E$ (not necessarily continuous), and the family $\left\{P_{A}: A \subset \mathbb{N}\right\}$ is a Boolean algebra of projections in $E$ (see Section 3). More generally, a subspace $F$ of $E$ is said to be monotone if it contains all the summands $E_{n}$ and is monotone with the induced M-decomposition $\left(P_{n} \mid F\right)$ (i.e., $P_{A}(F) \subset F$ for all $\left.A \subset \mathbb{N}\right)$.

We say that $\left(P_{n}\right)$ is a Schauder decomposition of $E$ if $x=\sum_{n=1}^{\infty} P_{n} x$ for every $x \in E$. A monotone Schauder decomposition is sometimes called a subseries Schauder decomposition. Spaces with Schauder decompositions are a generalization of AK-spaces. (In fact, Noll and Stadler [13] define AK-spaces as locally convex spaces having a suitable "system of sections" with "sectional convergence"; it is easy to see that their AK-spaces are precisely spaces with Schauder decompositions. On the other hand, Elstrodt and Roelcke [8] refer to monotone Schauder decompositions as "projection invariant" decompositions.)

If $\left(P_{n}\right)$ is a Schauder decomposition, then for every $u \in E^{\prime}$ one has

$$
\langle u, x\rangle=\sum_{n=1}^{\infty}\left\langle u, P_{n} x\right\rangle, \quad \text { for all } x \in E ;
$$

hence $u$ is determined by the sequence $\left(u_{n}\right) \in \prod_{n} E_{n}^{\prime}$, where $u_{n}:=u \mid E_{n}(n \in \mathbb{N})$. 
In general, whether we have a Schauder decomposition or merely an Mdecomposition, we define (see [17, p. 514] or [13, Section 2, p. 147]) the $\beta$-dual $E^{\beta}$ of $E$ as the subspace of $E^{*}$ consisting of all those functionals $u$ that can be written in the form

$$
\langle u, x\rangle=\sum_{n=1}^{\infty}\left\langle u_{n}, P_{n} x\right\rangle, \quad \text { for all } x \in E,
$$

for some sequence $\left(u_{n}\right) \in \prod_{n} E_{n}^{\prime}$. The functionals $u \in E^{\beta}$ for which the series in (*) converges absolutely for every $x \in E$ form a subspace called the $\alpha$-dual or Köthe dual of $E$ and denoted by $E^{\times}$. Finally, we denote by $E^{\phi}$ the space of all functionals $u$ of the form (*) that are determined by sequences $\left(u_{n}\right)$ with $u_{n}=0$ for large enough $n$. Wherever convenient, we tacitly identify $E^{\beta}, E^{\times}$, and $E^{\phi}$ with the corresponding defining subspaces of $\prod_{n} E_{n}^{\prime}$.

The following facts can be easily checked:

(a) $E^{\phi} \subset E^{\prime}$,

(b) $E^{\phi} \subset E^{\times} \subset E^{\beta} \subset E^{*}$,

(c) if $\left(P_{n}\right)$ is a Schauder decomposition, then $E^{\prime} \subset E^{\beta}$, and

(d) if $E$ is monotone, then $E^{\beta}=E^{\times}$.

Directly from the above definitions we have the following results.

PROPOSITION 1. If $E$ is a locally convex space with an $M$-decomposition $\left(P_{n}\right)$, then $E^{\phi}$ is sequentially dense in $\left(E^{\beta}, \sigma\left(E^{\beta}, E\right)\right)$. Hence, if $\left(P_{n}\right)$ is a Schauder decomposition, then

(a) $E^{\prime}$ is sequentially dense in $\left(E^{\beta}, \sigma\left(E^{\beta}, E\right)\right)$;

(b) $E^{\prime}=E^{\beta}$, provided that $\left(E^{\prime}, \sigma\left(E^{\prime}, E\right)\right)$ is sequentially complete.

Corollary 2. Let $E$ be a barrelled locally convex space with a Schauder decomposition $\left(P_{n}\right)$, and let $F$ be a subspace of $E$ containing all the summands $E_{n}$. Then $F$ is barrelled if and only if $\left(E^{\prime}, \sigma\left(E^{\prime}, F\right)\right)$ is sequentially complete. In this case $F^{\beta}=E^{\beta}=E^{\prime}$.

Proof. Apply Corollary 1 and Proposition 1.

This corollary comprises the first statement of Theorem 1. The next result is an extension of an analogous result for scalar sequence spaces (see [2, Proposition 3 (p. 55)] or $[11,4.2 .2($ p. 188)]).

Proposition 2. Let $E$ be a locally convex space with a monotone $M$-decomposition. If all the summands $E_{n}$ have weak ${ }^{*}$ sequentially complete duals $E_{n}^{\prime}$, then $\left(E^{\times}, \sigma\left(E^{\times}, E\right)\right)$ is also sequentially complete.

Proof. We have to show that $E^{\times}$is sequentially closed in $\left(E^{*}, \sigma\left(E^{*}, E\right)\right)$. Take a sequence $\left(u_{k}\right)$ in $E^{\times}$and assume that there is $u \in E^{*}$ such that $\lim _{k}\left\langle u_{k}, x\right\rangle=\langle u, x\rangle$ for all $x \in E$. For each $k \in \mathbb{N}$, let $u_{k}$ be determined by equality $(*)$ by a sequence $\left(u_{k n}\right)_{n \in \mathbb{N}} \in$ $\prod_{n} E_{n}^{\prime}$. Fix $x \in E$ and consider the finitely additive set functions $\mu_{k}, \mu: \mathscr{P}(\mathbb{N}) \rightarrow \mathbb{K}$ defined 
by the formulas $\mu_{k}(A)=\left\langle u_{k}, P_{A} x\right\rangle$ and $\mu(A)=\left\langle u, P_{A} x\right\rangle$. Since $\sum_{n=1}^{\infty}\left|\left\langle u_{k n}, P_{n} x\right\rangle\right|<\infty$ and

$$
\mu_{k}(A)=\sum_{n=1}^{\infty}\left\langle u_{k n}, P_{n} P_{A} x\right\rangle=\sum_{n \in A}\left\langle u_{k n}, P_{n} x\right\rangle,
$$

the functions $\mu_{k}$ are countably additive. By assumption, $\lim _{k} \mu_{k}(A)=\mu(A)$ for every $A \in \mathscr{P}(\mathbb{N})$, and so by Nikodym's countable additivity theorem $\mu$ is countably additive. Hence $\mu$ is determined by its values $\mu(\{n\})=\left\langle u, P_{n} x\right\rangle$; in particular,

$$
\langle u, x\rangle=\mu(\mathbb{N})=\sum_{n=1}^{\infty}\left\langle u, P_{n} x\right\rangle=\sum_{n=1}^{\infty}\left\langle v_{n}, P_{n} x\right\rangle,
$$

where $v_{n}:=u \mid E_{n}(n \in \mathbb{N})$. Note that the series above is absolutely convergent because $\mu$ is countably additive. Fix $n \in \mathbb{N}$. For every $x \in E_{n}$ we have $\lim _{k}\left\langle u_{k n}, x\right\rangle=\left\langle v_{n}, x\right\rangle$ for $x \in E_{n}$. Since for every $k \in \mathbb{N}$ we have $u_{k n} \in E_{n}^{\prime}$ and $E_{n}^{\prime}$ is weak* sequentially complete, it follows that $v_{n} \in E_{n}^{\prime}$.

Thus $u$ is determined via formula (*) by the sequence $\left(v_{n}\right) \in \prod_{n} E_{n}^{\prime}$, and so $u \in E^{\times}$.

COROLlaRY 3. Let $E$ be a locally convex space with a monotone Schauder decomposition $\left(P_{n}\right)$. Then $E$ is barrelled if and only if it is quasi-barrelled, each of its summands $E_{n}$ has a weak ${ }^{*}$ sequentially complete dual $E_{n}^{\prime}$, and $E^{\prime}=E^{\times}$.

Proof. Remembering that complemented subspaces of barrelled spaces are barrelled, it is enough to apply Fact A and Propositions 1 and 2.

From the last result and Fact B we obtain immediately the following corollary.

COROLlaRY 4. Let $E$ be a barrelled locally convex space with a Schauder decomposition $\left(P_{n}\right)$, and let $F$ be a monotone subspace of $E$. Then $F$ is barrelled if and only if $E^{\prime}=F^{\times}$.

The "moreover" part of Theorem 1 stated in the beginning of this section is simply a straightforward formulation of Corollary 4 . As a consequence of Corollary 4 we can derive the following extension of [13, Corollary 3] (their result does not include the case $p=1$ ).

EXAmple 1 . Let $(\Omega, \Sigma, \mu)$ be a $\sigma$-finite measure space and let $\left(\Omega_{n}\right)$ be a partition of $\Omega$ into a sequence of sets of finite measure. Fix $1 \leq p<\infty$. Then the projections $f \rightarrow f \chi_{\Omega_{n}}$. form a monotone Schauder decomposition of $L^{p}(\mu)$ with the corresponding summands $L_{n}^{p}(\mu)=\chi_{\Omega_{n}} . L^{p}(\mu)$. Applying Corollary 4 in this situation, we obtain the following result: a solid subspace $E$ of $L^{p}(\mu)$ containing all the subspaces $L_{n}^{p}(\mu)$ is barrelled if and only if whenever $g$ is a measurable function on $\Omega$ with $f . g \in L^{-1}(\mu)$ for all $f \in E$, then $g \in L^{q}(\mu)$ $\left(=L^{p}(\mu)^{\prime}=L^{p}(\mu)^{\times}\right)$, where $p^{-1}+q^{-1}=1$.

Remark 1. The above results (Corollaries 2, 3 and 4) contain, when written in the usual terminology for sequence spaces, namely as in (1) in Section 1, a result given by 
Swetits [18, Theorem 3.1] for the case when $\lambda$ is a Fréchet AK-space. His proof makes use of a theorem about inclusions between metrizable sequence spaces due to Bennett and Kalton [3, Theorem 1] that cannot be used in the more general situation of our result above. For this reason, the proofs are entirely different from each other. Also note than an example given in [18] shows that the hypothesis that $\lambda$ is an AK-space cannot be dropped.

An interesting situation that, as we shall see in a moment, has received particular attention is the following: for $A \subset \mathbb{N}$ and $n \in \mathbb{N}$, let

$$
d_{n}(A)=\frac{1}{n}|A \cap\{1,2, \ldots, n\}|=\frac{1}{n} \sum_{i=1}^{n} \chi_{A}(i) .
$$

If $\lim _{n} d_{n}(A)=0$, then the set $A$ is said to be of density zero. Let $\mathscr{Z}$ denote the family of all sets of density zero in $\mathbb{N}$. Clearly, $\mathscr{Z}$ is an ideal in the power set $\mathscr{P}(\mathbb{N})$. Now, let $\lambda$ be a monotone sequence space and consider the subspace $\mathscr{Z}(\lambda)$ (in some papers denoted by $\Sigma(\lambda)$ ) of all sequences in $\lambda$ that have their supports in $\mathscr{Z}$, i.e. those sequences of the form $\left(\alpha_{n} x_{n}\right)$, where $x=\left(x_{n}\right) \in \lambda$ and $\left(\alpha_{n}\right)$ is a sequence of zeros or ones $\lim _{n} \frac{1}{n} \sum_{j=1}^{n} \alpha_{j}$ $=0$. The definition of $\mathscr{Z}(\lambda)$ is due to Webb $[19$, p. 358] and it can be proved that $\mathscr{Z}(\lambda)$ is the union of the so-called scarce copies of $\lambda$ (see $[2$, Section 5 , Theorem 10] or $[11,4.3 .41])$. The space $\mathscr{Z}(\lambda)$ is also monotone and $(\mathscr{Z}(\lambda))^{\times}=\lambda^{\times}([11,4.2 .21],[13$, Lemma (p. 116)]) so that we can apply Corollary 4 above to obtain the following result.

Corollary 5. Let $\lambda$ be a monotone barrelled $A K$-space. Then $\mathscr{Z}(\lambda)$ is a barrelled subspace of $\lambda$.

Remark 2. The evolution of this result went as follows: Köthe [12, Section 27.1] stated it for $\lambda=l^{1}$. Webb [19, Lemma D.(1)] obtained it for perfect spaces in the sense of Köthe [12, Section 30]. For solid (or, which is the same in this case, monotone) Fréchet AK-spaces it appears in Bennett [1, Theorem 10]; see also Swetits [18, Corollary 3.4]. The next extension was Gupta and Kamthan's [11, 4.2.22] valid for solid AK-spaces. Noll and Stadler [13, Proposition 7] proved it for monotone (=solid) Banach AK-spaces with dual an AK-space (apparently not realizing that this case was already covered by Bennett's or Swetits' result). Finally, Elstrodt and Roelcke [8, Corollary 3.4 on p. 180], gave it in the above form. We think that the proof given here is much simpler than theirs. Concerning the scope of Corollary 5, some examples are given in [8, Sections 4 and 5] proving that it is not valid if one does not assume $\lambda$ to be monotone.

We are now going to extend Corollary 5 to the setting of spaces with Schauder decompositions. First, we prove an analogue of the equality $(\mathscr{Z}(\lambda))^{\times}=\lambda^{\times}$that was needed above to derive Corollary 5 from Corollary 4.

Proposition 3. Let $E$ be a locally convex space with a monotone Schauder decomposition $\left(P_{n}\right)$, and let

$$
\mathscr{Z}(E):=\left\{x \in E: x=P_{A} x, \text { for some } A \in \mathscr{Z}\right\} .
$$


Then

$$
\mathscr{Z}(E)^{\times}=E^{\times}
$$

Proof. We only have to show that $\mathscr{L}(E)^{\times} \subset E^{\times}$. Let $u \in \mathscr{L}(E)^{\times}$be determined via equality $(*)$ by a sequence $\left(u_{n}\right) \in \prod_{n} E_{n}^{\prime}$. Then $\sum_{n=1}^{\infty}\left|\left\langle u_{n}, P_{n} x\right\rangle\right|<\infty$ for all $x \in \mathscr{Z}(E)$, and we should verify that the same holds for all $x \in E$.

Suppose that for some $x \in E$ we have $\sum_{n=1}^{\infty}\left|\left\langle u_{n}, P_{n} x\right\rangle\right|=\infty$. Then, by [7, Theorem 1] or [13, Lemma on p. 116], there exists a set $A \in \mathscr{Z}$ such that $\sum_{n \in A}\left|\left\langle u_{n}, P_{n} x\right\rangle\right|=\infty$. But $x_{A}=P_{A}(x) \in \mathscr{Z}(E)$ and $\sum_{n \in A}\left|\left\langle u_{n}, P_{n} x\right\rangle\right|=\sum_{n=1}^{\infty}\left|\left\langle u_{n}, P_{n}\left(x_{A}\right)\right\rangle\right|<\infty$. A contradiction.

As an immediate consequence of Corollary 4 and Proposition 3, we obtain the version for Schauder decompositions of the main result in Elstrodt and Roelcke's paper [8, Theorem 3.1 on p. 176] (they work in the slightly more general setting of pseudodecompositions).

COROLlaRy 6. Let E be a barrelled locally convex space with a monotone Schauder decomposition $\left(P_{n}\right)$. Then $\mathscr{Z}(E)$ is a barrelled subspace of $E$.

In the following section we will see that Corollary 6 remains valid for arbitrary barrelled locally convex space with equicontinuous monotone M-decompositions that need not be Schauder decompositions.

\section{Barrelledness of spaces whose elements are of zero-density support.}

Definition. Let $\mathscr{R}$ be a ring of subsets of a set $S$. A (Boolean) $\mathscr{R}$-ring of projections in a locally convex space $E$ is a map $\mathbf{P}_{\mathscr{R}}$ that assigns to every $A \in \mathscr{R}$ a linear projection $P_{A}$ in $E$ so that the following conditions are satisfied:

(i) $P_{A \cap B}=P_{A} . P_{B}$ for all $A, B \in \mathscr{R}$.

(ii) $P_{A \cup B}=P_{A}+P_{B}$ for all disjoint $A, B \in \mathscr{R}$.

We will sometimes write $\mathbf{P}_{\mathscr{R}}=\left\{P_{A}: A \in \mathscr{R}\right\}$.

If $E$ is a locally convex space with a fixed ring of projections $\mathbf{P}_{\mathscr{R} R}$, then we define

$$
\mathscr{R}(E)=\left\{x \in E: P_{A}(x)=x \text { for some } A \in \mathscr{R}\right\} .
$$

Clearly, $P_{A}$ maps $E$ into $\mathscr{R}(E)$ for every $A \in \mathscr{R}$, and $\mathscr{R}(E)$ is a $\mathbf{P}_{\mathscr{R}}$-invariant subspace of $E$.

Monotone sequence spaces $\lambda$ are standard examples of spaces with a $\mathscr{P}(\mathbb{N})$-algebra of projections. In this case, of course, for every set $A \subset \mathbb{N}$ the linear projection $P_{A}$ in $\lambda$ is the map $x \mapsto \chi_{A} \cdot x=\left(\chi_{A}(n) x_{n}\right)$. More generally, as was already noted in Section 2, spaces with a monotone $\mathrm{M}$-decomposition have a natural $\mathscr{P}(\mathbb{N})$-algebra of projections. (However, there are $\mathscr{P}(\mathbb{N})$-algebras of projections that do not arise from M-decompositions; see Example 2 at the end of this paper.) Actually, what will really matter below is not the whole algebra of projections $\mathbf{P}_{\mathscr{P}(\mathbb{N})}=\left\{P_{A}: A \in \mathscr{P}(\mathbb{N})\right\}$, but its subring $\mathbf{P}_{\mathscr{T}}=\left\{P_{A}: A \in \mathscr{Z}\right\}$. Here, as in Section 2, $\mathscr{Z}$ denotes the ideal in $\mathscr{P}(\mathbb{N})$ consisting of all sets of density zero. 
The promised result generalizing Corollary 6 is Theorem 2 below. It can be applied to spaces like $l^{\infty}$ or $m_{0}$ (the space of sequences with finite range) that are barrelled but non-AK. Thus, as consequences of Theorem 2 , we have that the space $\mathscr{Z}\left(l_{\infty}\right)$, as well as the space $m_{0}(\mathscr{Z})=\mathscr{Z}\left(m_{0}\right)$ of all $\mathscr{Z}$-simple scalar functions on $\mathbb{N}$ (both with the sup-norm), are barrelled. (The latter fact follows also from Proposition 6 below.)

THEOREM 2. Let $E$ be a locally convex space with an equicontinuous ring of projections $\mathbf{P}_{\mathscr{Z}}=\left\{P_{A}: A \in \mathscr{Z}\right\}$. If $E$ is Banach-Mackey, or quasi-barrelled, or barrelled, then so is respectively, the subspace $\mathscr{Z}(E)$ of $E$.

In fact, as will be shown in Propositions 4 and 5, and Corollary 7 below, this result holds in the more general setting of rings with Property $(\mathrm{N})$, of which $\mathscr{Z}$ is a particular case (Proposition 6 below).

Let $\mathscr{R}$ be a ring of subsets of a set $S$. Denote by $b a(\mathscr{R})$ the Banach space of all bounded finitely additive scalar measures on $\mathscr{R}$ (equipped with the variation norm or with the equivalent sup-norm), and by $c a(\mathscr{R})$ its closed subspace consisting of countably additive measures. Following [16, p. 10], we say that the ring $\mathscr{R}$ has the Nikodym Property, or Property (N) for short, if every set $M \subset b a(\mathscr{R})$ that is pointwise bounded on $\mathscr{R}$ is uniformly bounded on $\mathscr{R}$. (That is, when $\sup \{|\mu(A)|: \mu \in M\}<\infty$ for all $A \in \mathscr{R}$ always implies that $\sup \{|\mu(A)|: \mu \in M, A \in \mathscr{R}\}<\infty)$. It is well-known that this property of $\mathscr{R}$ is equivalent to the barrelledness of the space $\operatorname{lin}\left\{\chi_{A}: A \in \mathscr{R}\right\}$ of all $\mathscr{R}$-simple scalar functions on $S$ equipped with the sup-norm. In particular, every $\sigma$-algebra has Property (N) $[6,1.3 .1]$.

Proposition 4. Let $E$ be a locally convex space with an equicontinuous ring of projections $\mathbf{P}_{\mathscr{L} R}$. Assume that the ring $\mathscr{R}$ has Property $(N)$ and that for every $A \in \mathscr{R}$ the subspace $P_{A}(E)$ of $E$ is Banach-Mackey. Then also the subspace $\mathscr{R}(E)$ is BanachMackey.

Proof. To simplify notation, write $X=\mathscr{R}(E)$. Suppose $X$ is not Banach-Mackey. Then there is a $\sigma\left(X^{\prime}, X\right)$-bounded sequence $\left(u_{n}\right)$ in $X^{\prime}$, and a bounded sequence $\left(x_{n}\right)$ in $X$, such that $\left|\left\langle u_{n}, x_{n}\right\rangle\right| \rightarrow \infty$, as $n \rightarrow \infty$.

Define a sequence $\left(\mu_{n}\right)$ of finitely additive scalar measures on $\mathscr{R}$ by $\mu_{n}(A)=\left\langle u_{n}\right.$, $\left.P_{A}\left(x_{n}\right)\right\rangle$. For every $n$, by the equicontinuity of $\mathbf{P}_{\mathscr{R}}$, the set $\left\{P_{A}\left(x_{n}\right): A \in \mathscr{R}\right\}$ is bounded in $X$; hence $\mu_{n}$ is a bounded measure.

Now, we verify that the sequence $\left(\mu_{n}\right)$ is pointwise bounded on $\mathscr{R}$. Let $A \in \mathscr{R}$. By the continuity of $P_{A}$, the sequence $\left(P_{A}\left(x_{k}\right)\right)$ is bounded in $P_{A}(E)$. But, by assumption, the subspace $P_{A}(E)$ is Banach-Mackey, hence

$$
\sup _{n}\left|\mu_{n}(A)\right| \leqslant \sup _{n, k}\left|\left\langle u_{n}, P_{A}\left(x_{k}\right)\right\rangle\right|<\infty .
$$

Since $\mathscr{R}$ has Property $(\mathrm{N})$, we conclude that the sequence $\left(\mu_{n}\right)$ is uniformly bounded on $\mathscr{R}$. However, by the definition of $X$, for every $n \in \mathbb{N}$ there is $A_{n} \in \mathscr{R}$ such that $x_{n}=P_{A_{n}}\left(x_{n}\right)$; hence $\left|\mu_{n}\left(A_{n}\right)\right|=\left|\left\langle u_{n}, x_{n}\right\rangle\right| \rightarrow \infty$, as $n \rightarrow \infty$, a contradiction.

Proposition 5. Let $E$ be a locally convex space with an equicontinuous ring of projections $\mathbf{P}_{\mathscr{R}}$. If $E$ is quasi-barrelled, then also its subspace $\mathscr{R}(E)$ is quasi-barrelled. 
Proof. Let $V$ be a bornivorous barrel in $\mathscr{R}(E)$. Recall that if $A \in \mathscr{R}$ then $P_{A}$ maps $E$ into $\mathscr{R}(E)$, and define

$$
U=\left\{x \in E: P_{A}(x) \in V \text { for all } A \in \mathscr{R}\right\}=\bigcap_{A \in \mathscr{R}} P_{A}^{-1}(V) .
$$

Since the projections $P_{A}, A \in \mathscr{R}$, are equicontinuous, it is clear that $U$ is a bornivorous barrel in $E$. But $E$ is quasi-barrelled; hence $U$ is a neighborhood of 0 in $E$. To finish, observe that $U \cap \mathscr{R}(E) \subset V$ so that $V$ is a neighborhood of 0 in $\mathscr{R}(E)$.

The following result is now a direct consequence of Propositions 4 and 5 , and Fact $A$.

COROLlaRY 7. Let $E$ be a barrelled locally convex space with an equicontinuous ring of projections $\mathbf{P}_{\mathscr{R}}$, where the ring of sets $\mathscr{R}$ has Property $(N)$. Then also the subspace $\mathscr{R}(E)$ of $E$ is barrelled.

We now return to Theorem 2 . In order to see that it is indeed a particular case of the above results, it suffices to prove the following proposition.

Proposition 6. The ring $\mathscr{Z}$ of all subsets of $\mathbb{N}$ of density zero has Property $(N)$.

We shall use the following three lemmas.

Lemma 1. For every sequence $\left(A_{k}\right)$ of infinite sets in $\mathscr{Z}$ there exist finite sets $F_{k} \subset A_{k}$ $(k \in \mathbb{N})$ such that $\bigcup_{k=1}^{\infty}\left(A_{k} \backslash F_{k}\right) \in \mathscr{Z}$.

Proof. There is a strictly increasing sequence $\left(n_{j}\right)$ in $\mathbb{N}$ with $n_{1}>1$ such that for $j \geqslant 1$

$$
d_{n}\left(A_{k}\right)<j^{-2}, \quad \text { for } 1 \leqslant k \leqslant j \text { and } n \geqslant n_{j} \text {. }
$$

For $j \geqslant 1$ set

$$
F_{j}=A_{j} \cap\left\{1,2, \ldots, n_{j}-1\right\} \quad \text { and } \quad B_{j}=A_{j} \backslash F_{j} \text {, }
$$

and let $B=\bigcup_{j=1}^{\infty} B_{j}$. Given $n \geqslant n_{1}$, there is $j \geqslant 1$ for which $n_{j} \leqslant n<n_{j+1}$. Then

$$
B \cap\{1,2, \ldots, n\}=\bigcup_{k=1}^{j} B_{k} \cap\{1,2, \ldots, n\} \subset \bigcup_{k=1}^{j} A_{k} \cap\{1,2, \ldots, n\}
$$

hence

$$
d_{n}(B) \leqslant \sum_{k=1}^{j} d_{n}\left(A_{k}\right)<j \cdot j^{-2}=j^{-1}
$$

It follows that $\lim _{n} d_{n}(B)=0$, i.e. $B \in \mathscr{Z}$.

Note. Lemma 1, as we discovered at the final stage of working on this paper, is closely related to some old results of Buck [4, Theorem 9 and Corollary on p. 573].

Lemma 2. (a) Let $M$ be a subset of $l^{1}$. Then $M$ is norm bounded if (and only if)

$$
\sup \left\{\left|\sum_{i \in A} x_{i}\right|: x \in M\right\}<\infty, \quad \text { for all } A \in \mathscr{Z} \text {. }
$$


(b) Let $M$ be a subset of $c a(\mathscr{P}(\mathbb{N})$ ). Then $M$ is uniformly bounded on $\mathscr{P}(\mathbb{N})$ if (and only if) it is pointwise bounded on $\mathscr{Z}$.

Proof. (a) The assumption means precisely that $M$ is $\sigma\left(l^{1}, \mathscr{L}\left(m_{0}\right)\right)$-bounded. Now, $\mathscr{Z}\left(m_{0}\right)$ is a monotone space and, by $[11,4.2 .21]$ or $[13$, Lemma (p. 116)] (or by Proposition 3 above), we have $\left(\mathscr{L}\left(m_{0}\right)\right)^{\times}=\left(m_{0}\right)^{\times}=l^{1}$. Therefore, $\mathscr{L}\left(m_{0}\right)$ is BanachMackey for the topology $\sigma\left(\mathscr{Z}\left(m_{0}\right), l^{1}\right)$, and it follows that $M$ is $\beta\left(l^{1}, \mathscr{Z}\left(m_{0}\right)\right)$-bounded. Finally, note that $\beta\left(l^{1}, \mathscr{Z}\left(m_{0}\right)\right)$ is the norm topology of $l^{1}$ because it is finer than $\beta\left(l^{1}, \phi\right)$ and coarser than $\beta\left(l^{1}, l^{\infty}\right)$, and these two are the norm topology on $l^{1}$.

In order to make Proposition 6 more accessible to measure theorists, we also sketch an alternative direct proof of (a). Suppose $M$ is not norm bounded. Then, as is easily seen, we can find a sequence $\left(x_{k}\right)$ in $M, x_{k}=\left(x_{k i}\right)$, and a sequence $\left(A_{k}\right)$ of finite subsets of $\mathbb{N}$ such that $\sup _{k}\left|\sum_{i \in A_{k}} x_{k i}\right|=\infty$. Now, following the pattern of the proof of [13, Lemma on p. 116] (with some obvious modifications), we can produce a strictly increasing sequence $(k(j))$ and sets $B_{j} \subset A_{k(j)}$ so that $\sup _{j}\left|\sum_{i \in B_{j}} x_{k(j) i}\right|=\infty$ and $B:=\bigcup_{j=1}^{\infty} B_{j} \in \mathscr{X}$. However, from the assumption on $M$ it is quite obvious that $\sup \left\{\left\|P_{A}(x)\right\|_{1}: x \in M\right\}<\infty$ for every $A \in \mathscr{Z}$. Thus we have arrived at a contradiction.

(b) Since $c a(\mathscr{P}(\mathbb{N}))$, equipped with the variation norm, is isometrically isomorphic to $l^{1},($ b) is simply a reformulation of (a).

In our next lemma, and in the proof of Proposition 6 that we give below, we are implicitly using the Yosida-Hewitt decomposition of measures $\mu \in b a(\mathscr{L})$ into the countably additive part $\mu^{c}$ and the purely finitely additive part $\mu^{p}$ (see [6, p. 30]).

Lemma 3. For every $\mu \in b a(\mathscr{Z})$ one has $\sum_{n=1}^{\infty}|\mu(\{n\})|<\infty$; hence the formula

$$
\mu^{c}(A)=\sum_{n \in A} \mu(\{n\})
$$

defines a countably additive scalar measure on $\mathscr{Z}$ (and even on $\mathscr{P}(\mathbb{N})$ ). Moreover, if a set $M \subset b a(\mathscr{L})$ is pointwise bounded on $\mathscr{Z}$, then also the set $M^{c}=\left\{\mu^{c}: \mu \in M\right\}$ is pointwise bounded on $\mathscr{Z}$.

Proof. The first part is obvious. The prove the "moreover" part, let $M \subset b a(\mathscr{Z})$ be pointwise bounded on $\mathscr{Z}$. Take any $A \in \mathscr{Z}$ and observe that $\mathscr{P}(A) \subset \mathscr{Z}$. Hence if $\mu \in M$, then

$$
\begin{aligned}
\left|\mu^{c}(A)\right| & \leqslant \sup \{|\mu(B)|: B \in \mathscr{P}(A)\} \\
& \leqslant \sup \{|\mu(B)|: \mu \in M, B \in \mathscr{P}(A)\}=: K,
\end{aligned}
$$

and $K<\infty$ because $\mathscr{P}(A)$, being a $\sigma$-ring, has Property $(\mathrm{N})$.

Proof of Proposition 6. Let $M$ be a pointwise bounded subset of $b a(\mathscr{Z})$; we have to show that $M$ is uniformly bounded on $\mathscr{Z}$. Define the subset $M^{c}$ of $c a(\mathscr{P}(\mathbb{N}))$ as in Lemma 3. According to Lemma $3, M^{c}$ is pointwise bounded on $\mathscr{Z}$. Hence, by Lemma $2, M^{c}$ is uniformly bounded on $\mathscr{Z}$. 
For every $\mu \in b a(\mathscr{Z})$ let $\mu^{p}:=\mu-\mu^{c}$ (the purely finitely additive part of $\mu$ ). It remains to verify that the set $M^{p}:=\left\{\mu^{p}: \mu \in M\right\}$ is uniformly bounded on $\mathscr{Z}$. Suppose this is false, so that we can find a sequence $\left(\mu_{n}\right)$ in $M$ and a sequence $\left(A_{n}\right)$ in $\mathscr{Z}$ such that $\left|\mu_{n}^{p}\left(A_{n}\right)\right| \rightarrow \infty$ as $n \rightarrow \infty$. Applying Lemma 1 , we find finite sets $F_{n} \subset A_{n}$ such that $B=\bigcup_{n=1}^{\infty} B_{n} \in \mathscr{Z}$, where $B_{n}=A_{n} \backslash F_{n}$. Now note that for each $\mu \in M$, its purely finitely additive part $\mu^{p}$ vanishes on all finite subsets of $\mathbb{N}$; hence $\left|\mu_{n}^{p}\left(B_{n}\right)\right|=\left|\mu_{n}^{p}\left(A_{n}\right)\right| \rightarrow \infty$, and $B_{n} \in \mathscr{P}(B) \subset \mathscr{L}$ for all $n$. Since $M^{p}$ is pointwise bounded on $\mathscr{P}(B)$ and $\mathscr{P}(B)$, as a $\sigma$-ring, has Property $(\mathrm{N})$, we have arrived at a contradiction.

REMARK 3. It is worth noting the following analogue of Lemma 3 for linear functionals.

Let $E$ be a locally convex space with an equicontinuous ring of projections $\mathbf{P}_{\mathscr{L}}$, and denote $P_{n}=P_{\{n\}}$ for $n \in \mathbb{N}$. Let $u \in E^{\prime}$. Then $\sum_{n=1}^{\infty}\left|\left\langle u, P_{n}(x)\right\rangle\right|<\infty$ for every $x \in E$, and the formula

$$
\left\langle u^{c}, x\right\rangle=\sum_{n=1}^{\infty}\left\langle u, P_{n}(x)\right\rangle
$$

defines an element $u^{c} \in E^{\prime}$.

Proof. If $x \in E$ then, by the equicontinuity of $\mathbf{P}_{\mathscr{X}}$, the set $\left\{P_{A}(x): A\right.$ a finite subset of $\mathbb{N}\}$ is bounded. It follows easily that $\sum_{n=1}^{\infty}\left|\left\langle u, P_{n}(x)\right\rangle\right|<\infty$. Thus the above formula makes sense. To see that $u^{c} \in E^{\prime}$ it is enough to observe that $\left\langle u^{c}, x\right\rangle=\lim _{n}\left(u \circ Q_{n}\right)(x)$, where $Q_{n}=P_{\{1,2 \ldots, n\}}$, and apply the equicontinuity of $\mathbf{P}_{\mathscr{T}}$ one more time.

REMARK 4. It appears to be still an open problem to find an intrinsic characterization of rings of sets with Property $(\mathrm{N})$. The same can be said about the Vitali-Hahn-Saks Property (VHS) and the Grothendieck Property (G); see [16] for the definitions of these properties and more information. As far as we know, one of the most recently given sufficient conditions for (VHS) $(=(N)+(G))$ is the following one introduced by Freniche [9]: a ring of sets $\mathscr{R}$ is said to have the Subsequential Interpolation Property (SI) if for every disjoint sequence $\left(A_{n}\right)$ in $\mathscr{R}$ and for every infinite set $M \subset \mathbb{N}$, there exist a set $A \in \mathscr{R}$ and an infinite $N \subset M$ such that $A_{n} \subset A$ if $n \in N$, and $A \cap A_{n}=\varnothing$ if $n \notin N$. Freniche himself provided in [10] an example showing that (VHS) does not imply (SI): namely, the algebra of those subsets of the unit interval that are simultaneously $F_{\sigma}$ and $G_{\delta}$ sets, considered earlier by Dashiell [5].

It is easy to see that also the ring $\mathscr{Z}$ fails to have (SI) although, as we have proved in Proposition 6 , it has $(\mathrm{N})$. Indeed, simply consider the sequence of sets $A_{n}:=\left\{2^{n}\right.$, $\left.2^{n}+1, \ldots, 2^{n+1}-1\right\} \in \mathscr{Z}$, and observe that none of its subsequences is covered by a set in $\mathscr{L}$. At this point it is worth noticing the following direct consequence of Lemma 1 (suggested to us by Z. Lipecki): the quotient ring $\mathscr{Z} / \mathscr{F}$, where $\mathscr{F}$ is the ideal of finite sets, has $(S I)$ ! (Hence, in the final part of the proof of Proposition 6 we could have used Freniche's VHS-type result from [9]; our direct argument was, however, much simpler.) 
Let us also observe that if we define (countably additive) measures $\mu_{n}$ on $\mathscr{Z}$ by $\mu_{n}(A)=2^{-n}\left|A \cap A_{n}\right|$, where $A_{n}$ are as above, then the resulting sequence $\left(\mu_{n}\right)$ is bounded in $b a(\mathscr{Z})$ and converges to zero pointwise, but is not uniformly exhaustive because $\mu_{n}\left(A_{n}\right)=1$ for each $n$. Thus $\mathscr{Z}$ does not have $(\mathrm{G})$.

Example 2. Let $E$ be the Banach space $b a(\mathscr{P}(\mathbb{N}))$. For $\mu \in E$ and $A \subset \mathbb{N}$ consider the measure $\mu_{A}$ given by $\mu_{A}(B):=\mu(A \cap B)$. Then $P_{A}(\mu)=\mu_{A}$ is a linear projection on $E$, and the set $\mathbf{P}=\left\{P_{A}: A \subset \mathbb{N}\right\}$ is an equicontinuous Boolean algebra of projections on $E$. By Theorem 2, the subspace $\mathscr{Z}(E)$ is barrelled.

Let now $F$ be the closed subspace of $b a(\mathscr{P}(\mathbb{N}))$ consisting of purely finitely additive measures (i.e., measures that vanish on the singletons). Then $F$ is invariant under the projections $P_{A}$ and, consequently, also the subspace $\mathscr{Z}(F)$ is barrelled. Note that for neither of these two spaces the projections $P_{n}=P_{\{n\}}$ form an M-decomposition.

Acknowledgments. This research was initiated while P. J. Paúl stayed at Clemson University (South Carolina) in the summer of 1990 . He wants to thank William H. Ruckle for his hospitality and fruitful discussions. The work was continued and essentially completed during the stay of L. Drewnowski at Universidad de Sevilla (Departamento de Matemática Aplicada) in the autumn of 1991. Financial support for these visits was provided by La Consejería de Educación y Ciencia de la Junta de Andalucía. The final version of the paper was written while $L$. Drewnowski held a visiting position at University of Mississippi in the spring semester of 1992. The authors wish also to thank Francisco J. Freniche (Universidad de Sevilla) for his valuable suggestions.

\section{REFERENCES} $17-30$.

1. G. Bennett, Some inclusion theorems for sequence spaces, Pacific J. Math. 46 (1973),

2. G. Bennett, A new class of sequence spaces with applications in summability theory, $J$. Reine Angew. Math. 266 (1974), 49-75. $511-524$.

3. G. Bennett and N. J. Kalton, Inclusion theorem for K-spaces, Canad. J. Math. 25 (1973),

4. R. C. Buck, The measure theoretic approach to density, Amer. J. Math. 68 (1946), $560-580$.

5. F. K. Dashiell, Non-weakly compact operators from order-Cauchy complete $C(S)$ lattices, with applications to Baire classes, Trans. Amer. Math. Soc. 266 (1981), 397-413.

6. J. Diestel and J. J. Uhl, Jr., Vector measures, Mathematical Surveys No. 15, (American Mathematical Society, Providence, RI, 1977).

7. R. Estrada and R. P. Kanwal, Series that converge on sets of null density, Proc. Amer. Math. Soc. 97 (1986), 682-686.

8. J. Elstrodt and W. Roelcke, Some dense barrelled subspaces of barrelled spaces with decomposition properties, Note Mat. 6 (1986), 155-203.

9. F. J. Freniche, The Vitali-Hahn-Saks theorem for Boolean algebras with the subsequential interpolation property, Proc. Amer. Math. Soc. 92 (1984), 362-366.

10. F. J. Freniche, Some classes of Boolean algebras related to the Vitali-Hahn-Saks and Nikodym property, Unpublished paper presented at a meeting in Oberwolfach in January, 1985.

11. P. K. Kamthan and M. Gupta, Sequence spaces and series, Lecture Notes in Pure and Applied Mathematics, Vol. 65, (Marcel Dekker, Inc., New York and Basel, 1981).

12. G. Köthe, Topological vector spaces I, (Springer-Verlag, 1969).

13. D. Noll and W. Stadler, Abstract sliding hump technique and characterization of barrelled spaces, Studia Math. 94 (1989), 103-120. 
14. P. Pérez Carreras and J. Bonet, Barrelled locally convex spaces, Notas de Matemática No. 131, (North-Holland, 1987).

15. W. H. Ruckle, Sequence spaces, Research Notes in Mathematics, Vol. 49, (Pitman, 1981).

16. W. Schachermayer, On some classical measure-theoretic theorems for non-sigma-complete Boolean algebras, Dissert. Math. 214 (1982), 1-33.

17. I. Singer, Bases in Banach spaces, II (Springer-Verlag, 1981).

18. J. Swetits, A characterization of a class of barrelled sequence spaces, Glasgow Math. J. 19 (1978), 27-31.

19. J. H. Webb, Sequential convergence in locally convex spaces, Proc. Cambridge Phil. Soc. 64 (1968), 341-364. 1978).

20. A. Wilansky, Modern methods in topological vector spaces (McGraw-Hill, New York,

L. Drewnowski:

INSTYTUT MATEMATYKI

UNIWERTSYTET A. MiCKIEWICZA

MATEJKI 48-49

60-769 POZNAŃ

Poland

and

Department of Mathematics

UNIVERSITY OF MISSISSIPPI

UNIVERSITY MS38677

U.S.A.

E-mail: DREWLECH@PLPUAM11.BITNET
M. Florencio and P. J. Paúl:

E.S. INGENIEROS INDUSTRIALES

Avda Reina Mercedes S/N

41012-SEVILLA

SPAIN

E-mail: PITI\%ETSII.US.ES@CERNVAX and PITI\%CICA.ES@CERNVAX 